\title{
The Frenet-Serret framework for aligning geometric curves
}

\author{
Nicolas J-B. BRUNEL ${ }^{1}$ and Juhyun PARK ${ }^{2}$ \\ 1 ENSIIE, 91025 Evry, France \\ nicolas.brunel@ensiie.fr \\ 2 Lancaster University, Lancaster, LA1 4YF, UK \\ juhyun.park@lancaster.ac.uk
}

\begin{abstract}
Variations of the curves and trajectories in 1D can be analysed efficiently with functional data analysis tools. In particular, the main sources of variations in $1 \mathrm{D}$ curves have been identified as amplitude and phase variations. Dealing with the latter gives rise to the problem of curve alignment and registration problems. It has been recognised that it is important to incorporate geometric features of the curves in developing statistical approaches to address such problems. Extending these techniques to multidimensional curves is not obvious, as the notion of multidimensional amplitude can be defined in multiple ways. We propose a framework to deal with the curve alignment in multidimensional curves as 3D objects. We propose a new distance between the curves that utilises the geometric information of the curves through the Frenet-Serret representation of the curves. This can be viewed as a generalisation of the elastic shape analysis based on the square root velocity framework. We develop an efficient computational algorithm to find an optimal alignment based on the proposed distance using dynamic programming.
\end{abstract}

Keywords: Curve Registration - Functional Data Analysis · FrenetSerret frames.

\section{Introduction}

We consider the general problem of aligning multidimensional curves as $3 \mathrm{D}$ objects. The curve alignement and registration problems are well studied for scalar curves (1D) under functional data analysis framework [4,7]. The richness of the registration problem comes from the variety of the criterion for comparing and measuring the similarity between the curves, which may also depend on the context. Nevertheless, in practice, good registration techniques "want" to align significant features of the curves, called landmarks, such as peaks and valleys, and more generally geometric patterns of the curves. Many statistical approaches have been developed to automate this process, without the need of manually identifying the landmarks. As the geometric information is contained in the derivatives, it is often better to align the curves based on the derivatives. 
A related problem is to identify the sources of variations, in particular, decoupling amplitude and phase variations has been the main framework to study the variations of $1 \mathrm{D}$ curves [3].

While the notion of amplitude is univocally defined for scalar curves, the generalisation to curves in Euclidean space $\mathbb{R}^{d}$ can be done in multiple ways [2]. Among possible approaches, the use of geometric features is shown to be effective for registering curves. This idea is formalised within the framework of elastic shape analysis [6], by considering significant landmarks and looking for invariant properties through group actions such as isometries or invariance by re-parametrisation. The basis of shape analysis is provided by the definition of proper spaces for representing objects, and the definition of an adapted distance. One of the successful applications of shape analysis for curves in general Euclidean spaces or more exotic ones is found with the use of the square root velocity transform (SRVT, [6]). The geometric feature is embedded in the first order derivative of the curves, the tangent of the curves.

In this article, we generalise the methodology based on the SRVT for the registration of two curves. Instead of using only the tangent information, we use an exhaustive description of the geometry of curves given by the so-called Frenet frame, which corresponds to the higher order information. This moving frame gives an explicit link to the complete geometric characterisation of a curve (curvature and torsion) through the Frenet-Serret formula. We propose a new distance between the curves based on the Frenet frame and demonstrates that the registration of the curves based on the Frenet frames is equivalent to stretching the curvatures and torsions. We show how to find an optimal solution using dynamic programming.

The article is organised as follows. In Section 2, we introduce the Frenet framework and review the square root velocity framework. Section 3 present our proposed methodology of curve alignment under the Frenet framework. Section 4 develops a computational algorithm.

\section{Preliminaries}

\subsection{Frenet-Serret Framework}

We consider regular curves $x$, i.e, functions such that the derivatives $x^{(k)}(\cdot), k=$ $0, \ldots 3$ exist, are continuous, and for all $t$ in $[0, T]$, we have $\dot{x}(t)=x^{(1)}(t) \neq 0$ and $\operatorname{det}\left(x^{(1)}(t), x^{(2)}(t), x^{(3)}(t)\right) \neq 0$. Consequently, we can write $x(t)=X(s(t))$ where $s \mapsto X(s)$ is the arclength parametrised curve and $t \mapsto s(t)$ is the curvilinear speed $\dot{s}(t)=\|\dot{x}(t)\|$ and $s(t)=\int_{0}^{t}\|\dot{x}(u)\| d u$. The length of the curve is $L=s(T)$. For clarity, we write $\frac{d}{d t} x=\dot{x}(t)$ for differentiation with respect to time and $\frac{d}{d s} X=X^{\prime}(s)$ for differentiation with respect to the curvilinear abscissa $s$.

The parametrised curve $\{s \mapsto X(s), s \in[0, L]\}$ is the geometric curve associated with $x$. For each $s \in[0, L]$, the tangent vector $T(s)=X^{\prime}(s)$ is normalised, and we can define additional normalised vectors $N$ and $B$ such that $N(s) \propto T^{\prime}(s)$ and $B(s) \propto N^{\prime}(s)$. Then, the matrix $Q(s)=[T(s)|N(s)| B(s)]$ is an orthonormal 
frame, which can be obtained by Gram-Schmidt orthonormalisation of the frame $\left[X^{\prime}(s)\left|X^{\prime \prime}(s)\right| X^{\prime \prime \prime}(s)\right]$. Quite remarkably, the Frenet frames are shown to be the solution of the following ODE:

$$
\left\{\begin{array}{l}
T^{\prime}(s)=\kappa(s) N(s), \\
N^{\prime}(s)=-\kappa(s) T(s)+\tau(s) B(s), \\
B^{\prime}(s)=-\tau(s) N(s),
\end{array}\right.
$$

where the functions $s \mapsto \kappa(s), \tau(s)$ are the curvature and torsion, respectively $(\kappa(s)>0)$. An alternative interpretation of this Frenet-Serret formula is that it defines an ODE in the Lie group $S O(p)$ as:

$$
\dot{Q}(s)=Q(s) A_{\theta}(s)
$$

where

$$
A_{\theta}(s)=\left[\begin{array}{ccc}
0 & -\kappa(s) & 0 \\
\kappa(s) & 0 & -\tau(s) \\
0 & \tau(s) & 0
\end{array}\right],
$$

is in the Lie algebra of skew-symmetric matrices, with the generalised curvature $\theta=(\kappa, \tau)$ and the initial condition $Q(0)=Q_{0}$.

The fundamental theorem of Differential Geometry of curves assures that two curves $x_{0}, x_{1}$ with the same $\theta$ (hence $L_{0}=L_{1}$ ) differ only by a translation $(a)$, a rotation $(O)$ and a reparametrisation $(h)$, i.e.,

$$
x_{1}(t)=a+O x_{0} \circ h(t) .
$$

Obviously this means that the Frenet frames $Q_{0}$ and $Q_{1}$ satisfy $Q_{0}(s)=O Q_{1}(s)$ for all $s \in[0, L]$. It is clear then that $Q_{i}$ and $\theta_{i}$ represents the shape of the curves $x_{i}$, for $i=1,2$, because they are invariant with respect to rigid Euclidean transformation, or to reparametrisation.

\subsection{Elastic Shape analysis}

Elastic shape analysis is a framework for comparing shapes by removing unnecessary and irrelevant variations between complex objects, typically Euclidean transformations and reparametrisation. It is also an efficient way of finding a good "time warping" function $h:[0, T] \longrightarrow[0, T]$ such that $x_{1}(h(t))=$ $X_{1}\left(s_{1}(h(t))\right)$ "looks like" $x_{0}(t)=X_{0}\left(s_{0}(t)\right)$. We denote the space of timewarping diffeomorphisms $h$ as $H_{T}$.

Curves are compared with a geodesic distance between them (defined through optimal deformations), which is based on the SRVT [6]. For each curve $x$, its square root velocity function is defined as

$$
q_{x}(t)=\frac{\dot{x}(t)}{\sqrt{\|\dot{x}(t)\|}}=\sqrt{\dot{s}(t)} T(s(t)),
$$

which can be viewed as a representation of the shape of the curve. The distance between two curves is then defined as the $L_{2}$ distance between $q_{x}$ and is parametrisation-independent. 
The SRVF transformation $F: x \mapsto \dot{x}(t) / \sqrt{\|\dot{x}(t)\|}$ helps defining a pre-shape space that is used for characterising the underlying shape of a given function. The pre-shape space for unit length open curves is $\mathcal{C}^{O}=\left\{q \in L^{2}\left([0, T], \mathbb{R}^{p}\right)\right\}$ and is simply the hypersphere of $L^{2}\left([0,1], \mathbb{R}^{p}\right)$. In order to align the curves $x_{0}, x_{1}$ with SRVF, we solve the following minimisation problem that defines at the same time a geodesic distance:

$$
d_{\text {srvf }}\left(x_{0}, x_{1}\right)=\inf _{O \in S O(3), h \in H_{T}} \int_{0}^{T}\left\|q_{0}(t)-O \sqrt{\dot{h}(t)} q_{1}(h(t))\right\|^{2} d t
$$

which can be written as

$$
\int_{0}^{T} \| \sqrt{\dot{s}_{0}(t)} T_{0}\left(s_{0}(t)\right)-\sqrt{\dot{s}_{1}(h(t)) \dot{h}(t)} O T_{1}\left(s_{1}(h(t)) \|_{2}^{2} d t .\right.
$$

The distance $d_{\text {srvf }}$ between two curves $x_{0}$ and $x_{1}$ is invariant to translation, rotation and re-parametrisation.

Exploiting this invariance, we can introduce the "space warping" diffeomorphism $\gamma:\left[0, L_{0}\right] \rightarrow\left[0, L_{1}\right]$ for any $h \in H_{T}$ such that $s_{1} \circ h=\gamma \circ s_{0}$ and define

$$
\mathcal{R}(O, \gamma)=\int_{0}^{L_{0}}\left\|T_{0}(s)-\sqrt{\dot{\gamma}(s)} O T_{1}(\gamma(s))\right\|_{2}^{2} d s .
$$

We introduce the function space $\Gamma_{S}$ of "space warping" diffeomorphisms, and the registration is obtained for $\left(O^{*}, \gamma^{*}\right)=\min _{\gamma, O} \mathcal{R}(O, \gamma)$ for $\gamma \in \Gamma_{S}$ and $\mathcal{R}\left(O^{*}, \gamma^{*}\right)$ is the elastic distance. In particular, the effective minimisation of $\mathcal{R}$ in $\gamma$ is done by dynamic programming.

\section{$3 \quad$ Elastic Shape Analysis and curvature stretching}

\subsection{Registration with Frenet-Serret frames}

We extend the registration of the curves within the Frenet-Serret framework. Specifically, our proposition is to find a "time warping" function $h:[0, T] \longrightarrow$ $[0, T]$ that tries to reduce the discrepancy between the moving frames $Q_{1}\left(s_{1}(h(t))\right)$ to $Q_{0}\left(s_{0}(t)\right)$. Similarly to the elastic distance, we propose the following distance between the curves

$$
D\left(x_{0}, x_{1}\right)=\int_{0}^{T} d\left(Q_{0}\left(s_{0}(t)\right), Q_{1}\left(s_{1}(t)\right)\right) \sqrt{\dot{s}_{0}(t) \dot{s}_{1}(t)} d t,
$$

where $d\left(Q_{0}, Q_{1}\right)$ is a distance between the frames in $S O(3)$. Standard choices are the Frobenius norm $\left\|Q_{0}-Q_{1}\right\|_{F}^{2}$ or the geodesic distance $\left\|\log Q_{1}^{\top} Q_{0}\right\|_{F}^{2}$, where $\log$ is the matrix logarithm. More generally, we can consider a distance based on the weighted norms such as $\|Q\|_{W, F}^{2}=\operatorname{Trace}\left(Q^{\top} W Q\right)$, indicating preferred directions in the frame. 
Introducing the "space warping" diffeomorphism $s_{1} \circ s_{0}^{-1}=\gamma \in \Gamma_{S}$ from $\left[0, L_{0}\right]$ to $\left[0, L_{1}\right]$ leads to

$$
D\left(x_{0}, x_{1}\right)=\int_{0}^{T} d\left(Q_{0}\left(s_{0}(t)\right), Q_{1}\left(\gamma\left(s_{0}(t)\right)\right)\right) \sqrt{\dot{s}_{0}(t) \frac{d}{d t}\left(\gamma \circ s_{0}\right)} d t .
$$

This can be expressed as

$$
D\left(x_{0}, x_{1}\right)=\int_{0}^{L_{0}} d\left(Q_{0}(s), Q_{1}(\gamma(s))\right) \sqrt{\gamma^{\prime}(s)} d s .
$$

The distance between curves can be seen as a weighted distance between the Frenet path $\mathcal{D}\left(Q_{0}, Q_{1} ; \gamma\right)=\int_{0}^{L_{0}} d\left(Q_{0}(s), Q_{1}(\gamma(s))\right) \sqrt{\gamma^{\prime}(s)} d s$. A direct extension of the distance $d_{\text {srvf }}$ is then the elastic Frenet-Serret distance

$$
D_{F S}\left(x_{0}, x_{1}\right)=\min _{h \in \Gamma_{T}, O \in S O(3)} D\left(x_{0}, O x_{1} \circ h\right) .
$$

We can also consider a distance that does not respect rotation invariance, but only reparametrisation, defined by

$$
D_{F S}^{0}\left(x_{0}, x_{1}\right)=\min _{h \in \Gamma_{T}} D\left(x_{0}, x_{1} \circ h\right) .
$$

As with elastic distance based on the SRVF, the registration problem is the computation of the distance function:

$$
\gamma_{0}^{*}=\arg \min _{\gamma \in \Gamma_{S}} \int_{0}^{L_{0}} d\left(Q_{0}(s), Q_{1}(\gamma(s))\right) \sqrt{\gamma^{\prime}(s)} d s .
$$

Then, the optimal "time warping" function for aligning $x_{1}(h(t))$ to $x_{0}(t)$ is given by $h_{0}^{*}=s_{1}^{-1} \circ \gamma_{0}^{*} \circ s_{0}$, where $\gamma_{0}^{*}$ is the optimal "space warping". Similarly, we can find the best reparametrisation and rotation $\left(\gamma^{*}, O^{*}\right)$ that solves the optimisation problem (1), and the curve $O^{*} x_{1}\left(h^{*}(t)\right)$ is aligned to $x_{0}(t)$ with $h^{*}=s_{1}^{-1} \circ \gamma^{*} \circ s_{0}$.

We note the "naive" distance $\int_{0}^{T} d\left(Q_{0}(s(t)), Q_{1}(\gamma(s(t)))\right) d t$ between the Frenet paths $Q_{0}$ and the warped one $Q_{1} \circ \gamma$ is not invariant with respect to reparametrisation by any $\gamma$ i.e., the distance $Q_{0} \circ \gamma$ and $Q_{1} \circ \gamma$ is different from the distance $Q_{0}$ and $Q_{1}$. It is worth noting that our elastic distance is not based on the $L^{2}$ distance between two SRVF $q_{x}$ that defines a sort of isometry between shapes, which decouples the distance between geometry (the tangent or the Frenet frame) and the influence of the arclength speed $\dot{s}(t)$.

Remark $1 D_{F S}^{0}$ is a direct generalisation of the standard elastic distance. If $d\left(Q_{0}, Q_{1}\right)=\left\|Q_{0}-Q_{1}\right\|_{F}^{2}=\left(\left\|Q_{0}\right\|_{F}^{2}+\left\|Q_{1}\right\|_{F}^{2}-2 \operatorname{Trace}\left(Q_{0}^{\top} Q_{1}\right)\right)$, the minimisation of $\int_{0}^{L_{0}} d\left(Q_{0}(s), Q_{1}(\gamma(s))\right) \sqrt{\gamma^{\prime}(s)} d s$ is then equivalent to the maximisation of $\int_{0}^{L_{0}}$ Trace $\left(Q_{0}^{\top}(s) Q_{1}(\gamma(s))\right) \sqrt{\gamma^{\prime}(s)} d s$. On the contrary, the minimisation of $\int_{0}^{L_{0}}\left\|T_{0}(s)-\sqrt{\gamma^{\prime}(s)} O T_{1}(\gamma(s))\right\|_{2}^{2} d s$ is equivalent the maximisation of $\int_{0}^{L_{0}} T_{0}^{\top}(s) T_{1}(\gamma(s)) \sqrt{\gamma^{\prime}(s)} d s$. This demonstrates that warping Frenet-Serret frames requires a higher degree of agreement between the geometries of $x_{0}$ and $x_{1}$. 


\subsection{Elastic distance and geometry stretching}

The previous section shows the alignment of $x_{0}, x_{1}$ is not done by changing the curvilinear speed but by changing the geometry of the curves. Indeed, elastic distance induces a specific family of transformations on the geometry of the curves. We have seen that the warping $\gamma_{0}^{*}$ defined in (3) permits to align (and transform) a curve of length $L_{1}$ to a curve of length $L_{0}$. More importantly, the space warping is a transformation of the geometry by stretching. For $Q_{0}$ : $\left[0, L_{0}\right] \longrightarrow S O(p)$ and $Q_{1}:\left[0, L_{1}\right] \longrightarrow S O(p)$, the two Frenet paths, the curves are stretched using a diffeomorphism $\gamma:\left[0, L_{0}\right] \longrightarrow\left[0, L_{1}\right]$. From section 2.1, the Frenet path $s \mapsto \tilde{Q}_{1}(s)=Q_{1}(\gamma(s))$ is also the solution of the following Frenet-Serret ODE:

$$
\begin{aligned}
\frac{d}{d s} \tilde{Q}_{1}(s) & =Q_{1}^{\prime}(\gamma(s)) \gamma^{\prime}(s), \\
& =Q_{1}(\gamma(s)) A_{\theta}(\gamma(s)) \gamma^{\prime}(s), \\
& =\tilde{Q}_{1}(s) A_{\tilde{\theta}}(s),
\end{aligned}
$$

where $\tilde{\theta}(s)=\theta(\gamma(s)) \gamma^{\prime}(s)$. The curve $X_{1}$ is stretched nonlinearly in order to look like the geometric curve similar to $X_{0}$, and $\tilde{\theta}$ is the corresponding stretched generalised curvature.

This is a "spatial" or geometric registration based on the family of deformations defined as $\theta \mapsto \gamma \cdot \theta=\gamma^{\prime} \theta \circ \gamma$, for any increasing diffeomorphism $\gamma$. This is a group action, i.e. for all $\gamma_{1}, \gamma_{2}$ diffeormophisms, and any generalised curvature $\theta$, we have

$$
\left(\gamma_{2} \circ \gamma_{1}\right) \cdot \theta=\gamma_{2} \cdot\left(\gamma_{1} \cdot \theta\right)
$$

Note that the stretching action does not permit to transform any geometry into another. Indeed, if $\theta_{0}$ and $\theta_{1}$ are two generalised curvatures such that the torsion $\tau_{0}>0$ and $\tau_{1}<0$, then we cannot find $\gamma$ such that $\gamma^{\prime} \tau_{1}(\gamma)=\tau_{0}$. The problem of finding a proper stretching $\gamma$ between $\theta_{0}$ and $\theta_{1}$ is somehow related to a boundary value problem of finding $\gamma$ such that $\theta_{1}(\gamma(s)) \gamma^{\prime}(s)=\theta_{0}(s)$ for all $s \in\left[0, L_{1}\right]$ with the constraint $\gamma(0)=0, \gamma\left(L_{0}\right)=L_{1}$ and $\gamma^{\prime}>0$. The registration problem gives an efficient way to characterise the proper stretching by solving a variation calculus problem

$$
\left\{\begin{array}{l}
\min _{\gamma} \int_{0}^{L_{0}} d\left(Q_{0}(s), Q_{1}(\gamma(s))\right) \sqrt{\gamma^{\prime}(s)} d s \\
\gamma(0)=0, \gamma\left(L_{0}\right)=L_{1} \\
\gamma^{\prime}>0
\end{array}\right.
$$

which can be solved with the corresponding Euler-Lagrange program. Instead, we introduce in the next section a dynamic programming algorithm that gives an efficient computation. But we should remark that the registration of two curves $x_{0}$ and $x_{1}$ with an elastic distance (based on SRVF or Frenet-Serret) is not equivalent to aligning curvatures and torsions. 


\section{Algorithm for pairwise alignment}

We introduce an iterative algorithm that computes the best space warping and rotation. It starts by finding $\gamma^{[0]}$, the minimum of (2), and then implements an alternative optimisation until convergence, i.e for $m \geq 0$

1. $O^{[m]}=\arg \min _{O} \mathcal{D}_{N}\left(Q_{0}, Q_{1} ; \gamma^{[m]}, O\right)$ by weighted averaging of rotations.

2. $\gamma^{[m+1]}=\arg \min _{\gamma} \mathcal{D}_{N}\left(Q_{0}, Q_{1} ; \gamma, O^{[m]}\right)$ by dynamic programming.

\subsection{Discretisation and dynamic programming}

We consider the problem of pairwise registration of two curves in $\mathbb{R}^{d}, s \mapsto$ $X_{0}(s), X_{1}(s)$ when these two curves are arc-length parametrised, with different lengths $L_{0}, L_{1}$, respectively. We start by considering only the criterion for finding the optimal stretching

$$
\mathcal{D}\left(Q_{0}, Q_{1} ; \gamma\right)=\int_{0}^{L_{0}} d\left(Q_{0}(s), Q_{1}(\gamma(s))\right) \sqrt{\gamma^{\prime}(s)} d s .
$$

We discretise the integral and use Dynamic Programming: we introduce points $s_{k}=\frac{k L_{0}}{N}, k=0, \ldots, N$ and define

$$
\mathcal{D}_{N}[\gamma]=\frac{L_{0}}{N} \sum_{k=0}^{N-1} g_{k}\left(x_{k}, u_{k}\right)+g_{N}\left(x_{N}\right)
$$

where $x_{k}=\gamma\left(s_{k}\right)$ is the current state, $u_{k}=\gamma^{\prime}\left(s_{k}\right)$ is the decision variable and

$$
g_{k}\left(x_{k}, u_{k}\right)=d\left(Q_{0}\left(s_{k}\right), Q_{1}\left(x_{k}\right)\right) \sqrt{u_{k}} .
$$

The terminal cost $g_{N}\left(x_{N}\right)$ is the usual terminal cost. In our case, it is not important as the final state is controlled and known, as $x_{N}=L_{1}$ should be satisfied. As we have $\gamma\left(s_{k+1}\right)=\gamma\left(s_{k}\right)+\frac{L_{0}}{N} \gamma^{\prime}\left(s_{k}\right)$, i.e., for $k \geq 0$,

$$
x_{k+1}=x_{k}+\frac{L_{0}}{N} u_{k} .
$$

We have then the following constraints on the state and control variables:

1. $x_{0}=0, x_{N}=L_{1}$ and for $k \in[1 \ldots N-1], 0<x_{k}<L_{1}$.

2. For $k=0, \ldots N-1, u_{k}>0$ and $x_{k}+\frac{L_{0}}{N} u_{k} \leq L_{1}$. In addition, we impose (for sparsity), that $u_{k} \leq \min \left(L_{1}-x_{k}, u^{\text {max }}\right)$.

3. $s_{k} \in\left\{\frac{k L_{0}}{N}, k=0, \ldots, N\right\}$.

The discretised criterion is $g_{k}(x, u)=d\left(Q_{0}\left(\frac{k L_{0}}{N}\right), Q_{1}(x)\right) \sqrt{u}$ for $k \leq N-1$ and $g_{N}(x)=\frac{L_{0}}{N} d\left(Q_{0}\left(L_{0}\right), Q_{1}\left(L_{1}\right)\right)$ if $x=L_{0}$ and $\infty$ if $x \neq L_{0}$. The state dynamics is $x_{k+1}=x_{k}+\frac{L_{0}}{N} u_{k}$, which gives the following algorithm: for every initial state $x_{0}$, the optimal cost is given by

$$
\left\{\begin{array}{l}
J_{N}\left(x_{N}\right)=g_{N}\left(x_{N}\right) \\
J_{k}\left(x_{k}\right)=\min _{u \in U_{k}\left(x_{k}\right)} g_{k}\left(x_{k}, u\right)+J_{k+1}\left(x_{k}+\frac{L_{0}}{N} u\right), k=0,1, \ldots, N-1 .
\end{array}\right.
$$


The optimal alignment function is given by the optimal path $s_{k} \mapsto x_{k}^{*}=\gamma^{*}\left(s_{k}\right)$, starting from $J_{0}\left(x_{0}\right)$, and taking the optimal configuration

$$
x_{k}^{*}=\arg \min _{x} J_{k}\left(x_{k}\right) .
$$

\subsection{Optimal rotation}

We consider now the estimation of the best rotation for minimising the divergence between the discretised aligned Frenet path from the previous stage:

$$
\min _{O \in S O(3)} \mathcal{D}_{N}\left(Q_{0}, Q_{1} ; \gamma^{*}, O\right)=\frac{L_{0}}{N} \sum_{k=0}^{N-1} d\left(Q_{0}\left(s_{k}\right), O Q_{1}\left(x_{k}^{*}\right)\right) \sqrt{u_{k}^{*}}
$$

The computation of the minimum depends on the type of the distance function used. For the standard Frobenius distance, the solution can be found by solving

$$
\min _{O} \sum_{k=0}^{N-1} \frac{L_{0} \sqrt{u_{k}^{*}}}{N} \operatorname{Trace}\left(Q_{1}\left(x_{k}^{*}\right) Q_{0}^{\top}\left(s_{k}\right) O\right)
$$

This has a closed-form solution, which is the polar part of the weighted mean $\sum_{k=0}^{N-1} \frac{L_{0} \sqrt{u_{k}^{*}}}{N} Q_{0}\left(s_{k}\right) Q_{1}^{\top}\left(x_{k}^{*}\right)$. If we use the geodesic distance, the solution is obtained by gradient descent in $S O(3)$, see $[5,1]$.

\section{References}

1. Le, H.: Estimation of riemannian barycentres. LMS Journal of Computation and Mathematics 7, 193-200 (2004)

2. Marron, J., Alonso, A.: Overview of object oriented data analysis. Biometrical Journal 56(5), 732-753 (2014)

3. Marron, J., Ramsay, J., Sangalli, L., Srivastava, A.: Functional data analysis of amplitude and phase variation. Statistical Science (2015)

4. Ramsay, J., Silverman, B.: Functional data analysis. Springer Series in Statistics, Springer, New York, second edn. (2005)

5. Rentmeesters, Q., Absil, P.A.: Algorithm comparison for karcher mean computations of rotation matrices and diffusion tensors. In: 19th European Signal Processing Conference (EUSPICO 2011) (2011)

6. Srivastava, A., Klassen, E.P.: Functional and shape data analysis. Springer (2016)

7. Wang, J.L., Chiou, J.M., Müller, H.G.: Functional data analysis. Annual Review of Statistics and Its Application 3, 257-295 (2016) 